This item was submitted to Loughborough's Research Repository by the author.

Items in Figshare are protected by copyright, with all rights reserved, unless otherwise indicated.

\title{
Effect of exercise intensity on circulating hepatokine concentrations in healthy men
}

PLEASE CITE THE PUBLISHED VERSION

PUBLISHER

NRC Research Press (C) The authors

VERSION

AM (Accepted Manuscript)

\section{PUBLISHER STATEMENT}

This paper was accepted for publication in the journal Applied Physiology, Nutrition, and Metabolism and the definitive published version is available at https://doi.org/10.1139/apnm-2018-0818.

\section{LICENCE}

CC BY-NC-ND 4.0

\section{REPOSITORY RECORD}

Willis, Scott, Jack A. Sargeant, Alice Thackray, Thomas E. Yates, David Stensel, Guruprasad P. Aithal, and James King. 2019. "Effect of Exercise Intensity on Circulating Hepatokine Concentrations in Healthy Men". figshare. https://hdl.handle.net/2134/36853. 
1 The effect of exercise intensity on circulating hepatokine concentrations in healthy men

2 Scott A Willis ${ }^{1,2}$, Jack A Sargeant ${ }^{1,2}$, Alice E Thackray ${ }^{1,2}$, Thomas Yates $^{2,3}$, David J Stensel ${ }^{1,2}$,

3 Guruprasad P Aithal ${ }^{4,5}$, James A King ${ }^{1,2}$.

4

$5{ }^{1}$ National Centre for Sport and Exercise Medicine, School of Sport, Exercise and Health

6 Sciences, Loughborough University, UK

$7 \quad{ }^{2}$ NIHR Leicester Biomedical Research Centre, UK

$8{ }^{3}$ Diabetes Research Centre, University of Leicester, UK

$9{ }^{4}$ Nottingham Digestive Diseases Centre, School of Medicine, University of Nottingham, UK

$10{ }^{5}$ NIHR Nottingham Biomedical Research Centre, Nottingham University Hospitals NHS

11 Trust and the University of Nottingham, UK

12

13 Authors' email addresses

14 Scott A Willis - $\underline{\text { S.Willis2@lboro.ac.uk }}$

15 Jack A Sargeant - js928@,leicester.ac.uk

16 Alice E Thackray - $\underline{\text { A.E.Thackray@,lboro.ac.uk }}$

17 Thomas Yates - ty20@1eicester.ac.uk

18 David J Stensel - D.J.Stensel@,1boro.ac.uk

19 Guruprasad P Aithal - Guru.Aithal@nottingham.ac.uk

20 James A King - J.A.King@,1boro.ac.uk

21

\section{Address for correspondence}

23 Dr James King

24 School of Sport, Exercise and Health Sciences

25 Loughborough University

1 
26 Leicestershire

27 United Kingdom

28 LE11 3TU

29 Phone: $+44(0) 1509228457$

30 Email: J.A.King@,lboro.ac.uk 


\section{Abstract}

32 Fibroblast growth factor 21 (FGF21), follistatin and leukocyte cell-derived chemotaxin 2 33 (LECT2) are novel hepatokines which are modulated by metabolic stresses. This study 34 investigated whether exercise intensity modulates the hepatokine response to acute exercise.

35 Ten young, healthy men undertook three 8-h experimental trials: moderate-intensity exercise $36\left(\mathrm{MOD} ; 55 \% \dot{V} \mathrm{O}_{2}\right.$ peak), high-intensity exercise (HIGH; $75 \% \dot{V} \mathrm{O}_{2}$ peak) and control (CON; 37 rest), in a randomised, counterbalanced order. Exercise trials commenced with a treadmill run 38 of varied duration to match gross exercise energy expenditure between trials (MOD vs HIGH; $2475 \pm 70$ vs $2488 \pm 58$ kJ). Circulating FGF21, follistatin, LECT2, glucagon, insulin, glucose and non-esterified fatty acids (NEFA) were measured before exercise and at $0,1,2,4$ and $7 \mathrm{~h}$ 41 post-exercise.

42 Plasma FGF21 concentrations were increased up to 4 h post-exercise compared to $\mathrm{CON}(P \leq$ $430.022)$ with greater increases observed at 1,2 and 4 h post-exercise during HIGH vs MOD $(P$ $44 \leq 0.025)$. Irrespective of intensity $(P \geq 0.606)$, plasma follistatin concentrations were elevated at 4 and $7 \mathrm{~h}$ post-exercise $(P \leq 0.053)$. Plasma LECT2 concentrations were increased immediately post-exercise $(P \leq 0.046)$ but were not significant after correcting for plasma 47 volume shifts. Plasma glucagon ( $1 \mathrm{~h} ; P=0.032)$ and NEFA (4 and $7 \mathrm{~h} ; P \leq 0.029)$ responses 48 to exercise were accentuated in HIGH vs MOD.

49 These findings demonstrate that acute exercise augments circulating FGF21 and follistatin. 50 Exercise-induced changes in FGF21 are intensity-dependent and may support the greater 51 metabolic benefit of high-intensity exercise.

52 Key words: exercise, physical activity, hepatokines, liver, FGF21, follistatin, LECT2, insulin 53 resistance 
55 The liver plays an integral role in the regulation of metabolic homeostasis through inter-organ 56 crosstalk with other metabolically active tissues (Iroz et al. 2015, Meex and Watt 2017). In 57 recent years, several exclusively or predominantly liver-secreted proteins, termed hepatokines, 58 have been identified as molecules possessing the capacity to exert potent metabolic effects, 59 both locally and distally (Meex et al. 2015). These include fibroblast growth factor 21 (FGF21), 60 shown to modulate glucose and lipid metabolism (Kharitonenkov et al. 2005, Potthoff et al. 61 2009, Xu et al. 2009, BonDurant and Potthoff 2018); follistatin, demonstrated to regulate 62 muscle growth (Amthor et al. 2004, Yaden et al. 2014) and influence pancreatic beta cell 63 function and survival (Zhao et al. 2015, Hansen et al. 2016a); and leukocyte cell-derived 64 chemotaxin 2 (LECT2), which can promote both skeletal muscle and adipose tissue insulin 65 resistance (Lan et al. 2014, Jung et al. 2018).

66 Chronic positive energy balance (Toye et al. 2007, Kirpich et al. 2011), hepatic steatosis (Meex 67 et al. 2015) and obesity-associated metabolic disease, modulate hepatic protein synthesis and 68 hepatokine secretion (Fu et al. 2012; Meex et al. 2015). Recent research suggests that 69 hepatokines may also be sensitive to acute perturbations in energy and substrate metabolism 70 through coordinating metabolic responses between the liver and extra-hepatic tissues (Xu et al. 71 2009, Lan et al. 2014, Hansen and Plomgaard 2016). For example, hepatokines are implicated 72 in the coordination of metabolic responses to both fasting and feeding (Lan et al. 2014, Markan 73 et al. 2014). Furthermore, hepatokines may participate in metabolic responses to exercise; 74 potentially contributing to the acute metabolic benefit associated with physical exertion such 75 as improved whole-body substrate metabolism and insulin sensitivity (Kim et al. 2013, Hansen et al. 2015, Weigert et al. 2018). 
77 To date, a handful of studies have examined the acute effects of exercise on circulating 78 hepatokines. This research has shown that exercise increases circulating levels of FGF21 and 79 follistatin (Hansen et al. 2011, Kim et al. 2013, Hansen et al. 2015, Hansen et al. 2016a, Hansen 80 et al. 2016b, Sargeant et al. 2018), whilst circulating LECT2 is suppressed after exercise in 81 mice (Lan et al. 2014) but is unaffected in humans (Sargeant et al. 2018). Exercise intensity is 82 a key variable which mediates the metabolic benefit of acute exercise, with protocols of higher intensity typically providing the greatest therapeutic effects (Wahren et al. 1978, Wen et al. 2011). Given that FGF21, follistatin and LECT2 responses to exercise are mediated via metabolic factors sensitive to exercise intensity (for example, glucagon, insulin and nonesterified fatty acids) (Hansen et al. 2015, Hansen et al. 2016a), it is possible that exercise intensity may also modulate circulating hepatokines.

One previous study explored this hypothesis, demonstrating that circulating FGF21 was higher in healthy men, one hour after 30 minutes of treadmill running at $80 \%$ vs $50 \%$ of maximum oxygen uptake (Kim et al. 2013). This study was, however, limited by the fact that the exercise protocols were not matched for energy expenditure and no control trial was included. Consequently, further research is required to explore the impact of exercise intensity on circulating hepatokines by matching exercise-related energy expenditure between exercise

94 protocols of different intensities; and to compare responses against a non-exercise control trial.

95 Therefore, the present study investigated the effect of exercise intensity, using two energy96 matched exercise trials, on circulating concentrations of FGF21, follistatin and LECT2 in 97 healthy men. Based on previous findings, it was hypothesised that both exercise intensities would lead to transient increases in circulating FGF21 and follistatin, whilst circulating LECT2 levels would be reduced following exercise. Furthermore, these responses would be more 100 pronounced after the performance of high- vs moderate-intensity exercise. 


\section{Participants}

103 Following approval from the Institutional Ethical Advisory Committee (ref: R16-P067), 10

104 healthy men participated in the study. Participant characteristics are presented in Table 1. All 105 participants were non-smokers, metabolically healthy, weight stable for three months prior to 106 participation ( $<2 \mathrm{~kg}$ body mass change) and were not currently taking any medications. Written 107 informed consent was provided by each participant before data collection commenced.

108

109

\section{Insert table 1}

\section{Participant pre-assessment}

112 Participants attended an initial pre-assessment session in which they completed a health screen

113 questionnaire, the Physical Activity Readiness Questionnaire (PAR-Q+; Bredin et al. 2013)

114 and food preference questionnaire, before undergoing anthropometric measurements. Height

115 and body mass were measured using a wireless measuring station (Seca 285, Seca Ltd,

116 Germany) and body mass index (BMI) was subsequently calculated. Waist circumference was

117 measured at the smallest circumference between the xiphoid process and the iliac crest, whilst

118 body composition was determined using bio-electrical impedance analysis (BC-418, TANITA,

119 Europe BC, Amsterdam, the Netherlands).

120 Participants then undertook a submaximal incremental exercise test on a motorised treadmill

121 (Technogym Excite Med, Cesena, Italy), consisting of four, 4-min stages at progressively faster speeds, to determine the relationship between treadmill speed and oxygen consumption $\left(\dot{\mathrm{V}} \mathrm{O}_{2}\right)$ 
123 for each participant. The first stage commenced at a speed between $7-8 \mathrm{~km} \cdot \mathrm{h}^{-1}$, with each

124 subsequent stage increasing by $1-2 \mathrm{~km} \cdot \mathrm{h}^{-1}$, according to participant fitness. Expired air was 125 sampled continuously using breath-by-breath indirect calorimetry (Cortex Metalyzer 3B, 126 Leipzig, Germany) to quantify $\dot{\mathrm{VO}}_{2}$ and carbon dioxide production $\left(\dot{\mathrm{V}} \mathrm{CO}_{2}\right)$. Heart rate was 127 recorded continuously using short-range telemetry (Polar T31; Polar Electro, Kempele, 128 Finland), whilst rating of perceived exertion (RPE; Borg. 1973) was assessed in the last $30 \mathrm{~s}$ 129 of each stage.

130 After a standardised rest period of $10 \mathrm{~min}$, participants then completed an incremental maximal 131 exercise test to determine peak oxygen uptake ( $\dot{\mathrm{VO}}_{2}$ peak). The test commenced at a gradient 132 of $0 \%$ and increased by $1 \%$ every minute until volitional exhaustion. The maximal exercise 133 test was designed to bring the participants to exhaustion within approximately 8-12 min and 134 the treadmill speed was determined by the individual performance of each participant in the 135 submaximal exercise test. The breath-by-breath data were analysed using a $30 \mathrm{~s}$ average 136 (Robergs et al. 2010) to identify the participants' $\dot{\mathrm{V}} \mathrm{O}_{2}$ peak.

\section{Experimental design}

138 Participants completed three main experimental trials: a moderate-intensity (MOD) trial, a 139 high-intensity $(\mathrm{HIGH})$ trial and a control $(\mathrm{CON})$ trial. The main trials were carried out in a 140 randomised, counterbalanced order, with at least five days separating each trial. In the $24 \mathrm{~h}$ 141 prior to each main trial, participants were asked to standardise their food intake using a weighed 142 food record and to abstain from exercise, caffeine and alcohol. Each main trial lasted $8 \mathrm{~h}$ $143(08: 00-16: 00)$ as shown schematically in Figure 1. 


\section{Experimental protocol}

148 On the day of the main trials, participants arrived at the laboratory at 08:00 following an

149 overnight fast of at least $10 \mathrm{~h}$. During MOD and HIGH trials, participants performed a 150 continuous treadmill run at moderate-intensity $\left(55 \% \dot{\mathrm{VO}}_{2}\right.$ peak $)$ and high-intensity $\left(75 \% \dot{\mathrm{VO}}_{2}\right.$ 151 peak), respectively, resting thereafter. Participants rested throughout the CON trial. Both 152 exercise protocols were designed to elicit a gross energy expenditure of $2510 \mathrm{~kJ}$ (600 kcal); 153 meaning the exercise duration differed between MOD and HIGH. The treadmill speed required 154 to elicit the target intensities was predicted via bivariate linear regression using participants' $155 \dot{\mathrm{V}} \mathrm{O}_{2}$ peak and $\dot{\mathrm{V}} \mathrm{O}_{2}$-running speed relationship determined in the pre-assessment session. The rate of energy expenditure for each trial was then calculated using equations by Frayn (1983)

157 and the exercise duration required to induce a gross energy expenditure of $2510 \mathrm{~kJ}$ was 158 subsequently estimated. Expired air was sampled continuously during exercise to determine 159 the actual gross energy expenditure and percentage substrate oxidation of the exercise period.

160 The speed of the treadmill was adjusted, if necessary, to maintain the target $\mathrm{V}_{2}$. Heart rate 161 was monitored continuously throughout; whilst RPE was recorded every 10 min. Exercise commenced between 08:00 and 09:00 in a staggered fashion so that exercise finished at 09:00.

163 A standardised breakfast (jam sandwich with butter, strawberry breakfast bar, banana and 164 chocolate milkshake) was provided to the participants at $2 \mathrm{~h}$ post-exercise (11:00), containing 165 an energy content equal to $35 \%$ of the participants' estimated daily energy requirement and a 166 macronutrient content of $69 \%$ carbohydrate, 14\% protein and $17 \%$ fat. Individuals' daily 167 energy requirements were calculated from their estimated basal metabolic rate (BMR), which was multiplied by a physical activity correction factor of 1.4 (Mifflin et al. 1990) to reflect the sedentary nature of prolonged sitting in the laboratory during the main trials. A standardised 8 
170 lunch (cheese sandwich with butter, salted crisps, mini chocolate roll and apple) was then

171 provided at 6 h post-exercise (15:00), also containing $35 \%$ of the participants' estimated daily

172 energy requirement ( $42 \%$ carbohydrate, $13 \%$ protein and $45 \%$ fat).

\section{Blood sampling}

174 Venepuncture blood samples were taken from an antecubital vein upon arrival (baseline; 08:00)

175 and immediately after exercise (0 h; 09:00). At approximately $0.5 \mathrm{~h}$, a cannula (Venflon,

176 Becton Dickinson, Helsingborg, Sweden) was inserted into an antecubital vein for further

177 venous blood sample collection at 1, 2, 4 and $7 \mathrm{~h}$ post-exercise. Following each collection, the

178 cannula was flushed with $10 \mathrm{~mL}$ of saline $(0.9 \% \mathrm{w} / \mathrm{v}$ sodium chloride, Fannin Ltd, Dublin,

179 Ireland) to maintain patency. To avoid dilution of subsequent samples, residual saline was

180 drawn off immediately prior to each sample collection using a $2 \mathrm{~mL}$ syringe. All blood samples

181 were taken with the participant in a semi-supine position to control for any postural changes in

182 plasma volume.

183 Blood samples were collected into pre-chilled EDTA and lithium heparin monovettes (Sarstedt,

184 Leicester, UK). All monovettes were centrifuged at $1750 \mathrm{~g}$ for $10 \mathrm{~min}$ at $4^{\circ} \mathrm{C}$ (Heraeus

185 Labofuge 400R, Thermo Electron, Osterode, Germany) and the resultant plasma supernatant

186 was aliquoted into cryovials. All samples were stored at $-80^{\circ} \mathrm{C}$ until subsequent (batch) analysis.

187 Haemoglobin and haematocrit concentrations were determined from each sample using $20 \mu \mathrm{L}$

188 micropipettes and $20 \mu \mathrm{L}$ heparinised microhaematocrit tubes. These measures were taken in

189 duplicate and triplicate, respectively; and were used for subsequent estimation of changes in 190 plasma volume (Dill and Costill 1974).

191 Biochemical analyses 
192 Commercially available enzyme-linked immunosorbent assays were used to determine plasma 193 concentrations of FGF21(DF2100, R\&D Systems, Oxford, UK), follistatin (DFN00, R\&D 194 Systems, Oxford UK), LECT2 (5327, MBL International, Massachusetts, USA), insulin (10195 113-01, Mercodia AB, Uppsala, Sweden) and glucagon (10-1271-01, Mercodia AB, Uppsala, 196 Sweden). Samples from each participant were analysed on the same assay plate to eliminate 197 inter-assay variation. The mean within-batch coefficients of variation (CVs) for these assays 198 was $\leq 6.0 \%$. Plasma glucose (A11A01668, Horiba Medical, Montpellier, France) and non199 esterified fatty acids (NEFA; FA115, Randox Laboratories Ltd., County Antrim, UK) were 200 determined using enzymatic, colorimetric methods using a bench-top analyser (Pentra 400; 201 Horiba Medical, Montpellier, France). The mean within-batch CVs for plasma glucose and 202 NEFA were $0.43 \%$ and $1.95 \%$, respectively.

\section{Sample size calculation}

204 Based on published data by our research group examining the effects of moderate-intensity 205 exercise on the total area under the curve (AUC) for FGF21 in normal-weight healthy men 206 (mean difference \pm SD: $286 \pm 340 \mathrm{pg} \cdot \mathrm{mL}^{-1} \cdot 7 \mathrm{~h}^{-1}$, giving a standardised difference of 0.84 , and 207 with an intra-person correlation of 0.76) (Sargeant et al. 2018), we required nine participants 208 to complete our three-trial cross-over study to detect a main effect of trial for FGF21 total AUC, 209 with $90 \%$ power and an alpha error rate of 0.05 . To account for participant drop-out or non210 compliance, 10 individuals were recruited. Notably, based on similar data from our previous 211 study for follistatin total AUC (mean difference \pm SD: $630 \pm 942 \mathrm{pg} \cdot \mathrm{mL}^{-1} \cdot 7 \mathrm{~h}^{-1}$, giving a 212 standardised difference of 0.67 , and with an intra-person correlation of 0.88 ), we also had over $21390 \%$ power to detect a main effect of trial on follistatin total AUC in the current study.

\section{Statistical analyses}


215 All statistical analyses were performed using SPSS version 23 (SPSS Inc., Chicago, Illinois).

216 The preprandial (baseline-2 h), postprandial (2-7 h) and total AUC were calculated using the

217 trapezoidal method for all outcomes during each trial. Shapiro-Wilk tests were used to assess

218 the distribution of the data and, when not normally distributed, data were log transformed and

219 reassessed. Differences between exercise characteristics were assessed using paired $t$-tests.

220 One-way repeated-measures analysis of variance (ANOVA) was used to examine differences

221 between trials in baseline concentrations and AUC values for each analyte. Two-way, repeated-

222 measures ANOVA (within-participant factors: trial and time) was used to assess differences in

223 hepatokine and metabolite responses over the duration of the different trials. Where a

224 significant trial and interaction effect was observed, post-hoc analyses were performed using

225 one-way ANOVAs and subsequent pairwise comparisons at each time point to locate any

226 differences. A Bonferroni adjustment for multiple comparisons was then applied. If Mauchly's

227 test of sphericity was violated, Greenhouse-Geisser corrected values were used. Correction of

228 FGF21, follistatin, glucose, NEFA, insulin and glucagon data for plasma volume changes did

229 not alter the statistical significance of findings, therefore unadjusted values are presented. The

230 statistical outcomes for LECT2 were altered by plasma volume adjustment, therefore statistical

231 outcomes are presented for both adjusted and unadjusted data. An alpha value of $P<0.05$ was

232 set to indicate statistical significance. Data are presented as means \pm SD or SEM, where

233 indicated. 


\section{Exercise characteristics}

236 The characteristics for the MOD and HIGH exercise protocols are presented in Table 2.

237 Treadmill speed, oxygen uptake, and relative exercise intensity were greater during HIGH 238 compared to MOD (all $P \leq 0.001$ ). However, the exercise duration was shorter during HIGH 239 than $\operatorname{MOD}(P<0.001)$; resulting in similar gross energy expenditures between the two exercise 240 protocols $(P=0.459)$. Respiratory exchange ratio $(\mathrm{RER})$ and percentage carbohydrate 241 oxidation were significantly higher, and percentage fat oxidation significantly lower, in HIGH 242 compared with MOD (all $P \leq 0.001$ ). Heart rate and RPE were also greater in HIGH in 243 comparison to MOD (both $P \leq 0.001$ ).

244

245

Insert table 2

\section{Hepatokine responses to exercise}

248 Baseline plasma concentrations of FGF21 were similar between trials $(P=0.235)$. Two-way 249 ANOVA showed a significant main effect of trial and trial by time interaction for plasma 250 FGF21 concentrations (both $P<0.001$; Figure 2A). Post-hoc analyses revealed significantly 251 higher plasma FGF21 concentrations during MOD compared to $\mathrm{CON}$ at 0,1 and $2 \mathrm{~h}$; and during 252 HIGH compared to $\mathrm{CON}$ at 1,2 and $4 \mathrm{~h}$ (all $P \leq 0.017$ ). Additionally, plasma FGF21 253 concentrations were significantly higher during HIGH vs MOD at 1,2 and $4 \mathrm{~h}$ (all $P \leq 0.025$ ).

254 Table 3 details the preprandial (baseline-2 h), postprandial (2-7 h) and total FGF21 AUC. 
255 Compared with control, the FGF21 AUC was higher preprandially in MOD and HIGH $(P \leq$ $2560.009)$ yet remained elevated postprandially only in $\operatorname{HIGH}(P=0.001)$.

257 Boxplot analysis of total trial AUC values for plasma follistatin identified one participant as an 258 outlier (Field 2009). This was due to the participant exhibiting mean plasma follistatin 259 concentrations during HIGH which were 13 times greater than the remaining group SD on the 260 same trial. Consequently, all data for this participant were removed from follistatin analyses 261 and thus results are presented for $n=9$. No differences were observed in fasting plasma 262 follistatin concentrations between trials at baseline $(P=0.138)$. Two-way ANOVA showed a 263 significant main effect of trial, time and trial by time interaction for plasma follistatin concentrations (all $P \leq 0.002$; Figure 2B). Post-hoc analyses revealed that when compared to 265 $\mathrm{CON}$, plasma follistatin concentrations tended to be higher at $4 \mathrm{~h}(P=0.053)$ and were 266 significantly higher at $7 \mathrm{~h}$ during $\operatorname{MOD}(P=0.007)$. Furthermore, plasma follistatin 267 concentrations were significantly higher at 4 and 7 h during HIGH compared to CON (both $P$ $268 \leq 0.003)$. Examination of AUC data showed that circulating follistatin concentrations were 269 greater in HIGH vs CON postprandially $(P=0.003$; Table 3$)$.

270 Baseline plasma concentrations of LECT2 did not differ between trials $(P=0.108)$. Two-way 271 ANOVA showed a significant main effect of time and trial by time interaction for plasma 272 LECT2 concentrations (both $P \leq 0.004$; Figure 2C). Post-hoc analyses identified elevated 273 plasma LECT2 concentrations immediately post-exercise during MOD and HIGH trials 274 compared to CON (both $P \leq 0.046$ ), with a similar response between exercise trials; however, 275 following adjustment for plasma volume changes across the trials, statistical significance did 276 not remain (both $P \geq 0.243$ ). No differences in AUC values were observed between trials (Table $2773)$. 


\section{Hormone and metabolite responses to exercise}

284 Plasma hormone and metabolite responses at baseline and after exercise are shown in Figure 3.

285 Baseline plasma concentrations of glucose, NEFA and glucagon were not statistically different 286 between trials (all $P \geq 0.124$ ). However, plasma insulin was significantly higher and the 287 glucagon to insulin ratio was significantly lower at baseline in MOD compared to CON $(P \leq$ 0.01). In response to exercise, two-way ANOVA showed a significant main effect of time and trial by time interaction for glucose and NEFA (all $P \leq 0.001$ ), whilst a significant main effect of trial was also observed for NEFA $(P<0.001)$. Post-hoc analyses revealed significantly higher plasma NEFA concentrations during MOD compared to CON at 0 and $2 \mathrm{~h}$; and during HIGH compared to CON at $0,2,4$ and 7 h (all $P \leq 0.044$ ). Additionally, plasma NEFA concentrations were significantly higher during $\mathrm{HIGH}$ in comparison to MOD at 4 and $7 \mathrm{~h}$ 294 (both $P \leq 0.029$ ). No significant differences in plasma glucose were found between trials at any 295 time point $(P \geq 0.084)$.

296 There was a significant main effect of time and trial by time interaction for glucagon, insulin 297 and glucagon to insulin ratio (all $P \leq 0.002$ ), whilst a significant main effect of trial was also 298 observed for glucagon $(P<0.001)$. Post-hoc analyses revealed that when compared to CON, 299 plasma glucagon concentrations were significantly higher at 0,1 and $2 \mathrm{~h}$ during MOD and $300 \mathrm{HIGH}$ (all $P \leq 0.045$ ). Furthermore, plasma glucagon concentrations were significantly higher 301 at $1 \mathrm{~h}(\mathrm{P}=0.032)$ and tended to be higher at $2 \mathrm{~h}(P=0.061)$ during HIGH compared to MOD. 14 
302 Subsequently, a significantly greater glucagon to insulin ratio was observed at $1 \mathrm{~h}$ during MOD, 303 and at 1 and $2 \mathrm{~h}$ during HIGH, when compared to CON (all $P \leq 0.006$ ). However, no differences 304 were observed between the two exercise trials $(P \geq 0.656)$. No significant differences in plasma 305 insulin were found between trials at any time point post-exercise $(P \geq 0.378)$.

306

307

Insert figure 3 
309 The present study explored the acute effect of exercise intensity on circulating concentrations

310 of FGF21, follistatin and LECT2 in a population of healthy men. Our novel observations are

311 that high-intensity exercise elicited a greater post-exercise increase in circulating FGF21 when

312 compared to moderate-intensity exercise. Furthermore, circulating follistatin was elevated after

313 exercise independently of exercise intensity; whilst LECT2 appeared not to respond to acute

314 bouts of exercise.

315 FGF21 is regarded as a metabolically beneficial hepatokine which is upregulated by obesity 316 and obesity-related diseases (Chavez et al. 2009, Dushay et al. 2010), potentially as a 317 compensatory mechanism to preserve metabolic homeostasis (Li et al. 2018). FGF21 has also 318 been shown to be acutely responsive to metabolic stresses such as fasting, protein restriction 319 and exercise (Galman et al. 2008, Kim et al. 2013, Laeger et al. 2014). The findings from the 320 present study demonstrate that the FGF21 response to acute exercise is intensity dependent, 321 with a greater response evident after an isoenergetic bout of high- vs moderate-intensity 322 exercise. This finding is supported by previous observations suggesting that greater post323 exercise increases in circulating FGF21 may be present with increasing exercise intensity (Kim et al. 2013). It must be noted, however, this previous study failed to isolate the effect of exercise intensity from the confounding influence of exercise-related energy expenditure (Kim et al. 326 2013). Our findings also demonstrate that the exercise-induced increase in circulating FGF21 327 persisted for up to four hours post-exercise which is consistent with previous reports from our 328 laboratory (Sargeant et al. 2018) and elsewhere (Kondo et al. 2011).

329 Although it is known that exercise enhances the expression and secretion of FGF21 from the 330 liver (Kim et al. 2013, Hansen et al. 2015), the mechanisms underpinning the exercise intensity331 dependent increase in circulating FGF21 cannot be delineated from the present study. Our 16 
results cannot distinguish whether the identified difference in systemic FGF21 was due to

333 altered protein expression, secretion or clearance. Our data may, however, indicate an

334 influential role of glucagon. Specifically, whilst no intensity-dependent differences were

335 apparent in circulating NEFA, insulin or the glucagon to insulin ratio (all of which have been

336 shown to modulate hepatic FGF21 secretion; Inagaki et al. 2007, Arafat et al. 2013, Hansen et

337 al. 2015, Hansen et al. 2016b), circulating glucagon was two-fold higher in response to high-

338 vs moderate-intensity exercise. It is therefore possible that the greater FGF21 response to high-

339 intensity exercise was mediated by a relatively larger glucagon response. This possibility is

340 supported by preclinical data which has demonstrated an independent role of glucagon as a

341 stimulus of hepatic FGF21 expression and secretion (Arafat et al. 2013, Habegger et al. 2013,

342 Cyphert et al. 2014, Alonge et al. 2017, Kim et al. 2018); however, the descriptive nature of

343 the present study prevents us from unpicking this further.

344 It has been suggested that exercise-related augmentation of FGF21 may contribute to the

345 beneficial metabolic effects of exercise (Hansen et al. 2016b, Weigert et al. 2018). Notably, in

346 Wistar rats, Loyd et al (2016) recently demonstrated that FGF21 is essential for the beneficial

347 effects of exercise training on hepatic steatosis and glycaemic control to be realised during a

348 period of high-fat feeding. In the present study, the physiological relevance of the greater, yet

349 transient augmentation of circulating FGF21 in response to high- vs moderate-intensity

350 exercise, is not clear. It is possible that the enhanced response may contribute to the greater

351 metabolic benefits associated with high-intensity exercise, including greater improvements in

352 insulin sensitivity and glycaemic control (Hayashi et al. 2005, Rynders et al. 2014); or it may

353 reflect a participatory role of the protein in the metabolic response to exercise and recovery.

354 Carefully designed experiments utilising animal models are required to shed more light on this 355 question. 
356 Previous research has identified follistatin as another hepatokine which is transiently increased

357 in the circulation in response to a single bout of exercise (Hansen et al. 2011, Hansen et al.

358 2016a, Hansen et al. 2016b, Sargeant et al. 2018). In the present study, both exercise intensities

359 led to a similar increase in circulating follistatin concentrations. Previous studies have shown

360 that the circulating glucagon to insulin ratio is a potent stimulus of hepatic follistatin release in

361 response to exercise (Hansen et al. 2016a, Hansen et al. 2016b). The comparable follistatin

362 response between exercise intensities may therefore be due to equivalent changes in the

363 circulating glucagon to insulin ratio. The importance of these gluco-regulatory hormones may

364 help explain recent findings showing that circulating follistatin was elevated after both high-

365 intensity interval exercise and resistance exercise; but not low-to-moderate intensity

366 continuous exercise (Perakakis et al. 2018). Although not measured in this previous study, it is

367 likely that only the high-intensity exercise modalities would have triggered an increase in

368 circulating glucagon and/or a decrease in circulating insulin; as opposed to the low-to-moderate

369 intensity exercise (Wolfe et al. 1986, Lira et al. 2012).

370 Whilst the physiological relevance of the follistatin response to exercise is not fully understood,

371 follistatin has been identified to play a beneficial role in several metabolic actions which may

372 be relevant to exercise. This includes promoting skeletal muscle growth and preventing

373 degradation through its actions as a natural antagonist of myostatin (Amthor et al. 2004, Yaden

374 et al. 2014). Moreover, chronic overexpression of follistatin promotes pancreatic $\beta$-cell

375 survival (Zhao et al. 2015, Hansen et al. 2016a), whilst acute administration of follistatin may

376 reduce glucagon secretion from human islets in a potential negative feedback loop (Hansen et

377 al. 2016a). Therefore, an exercise-induced increase in circulating follistatin may contribute in

378 some capacity to the positive metabolic effects of exercise; however, the exact role needs to be

379 delineated further. 
When interpreting the follistatin results in the present study, one important methodological consideration warranting recognition was the decision to feed participants after exercise. In previous studies, follistatin responses to exercise have been examined with study participants remaining fasted throughout experimental trials (Hansen et al. 2011; Hansen et al. 2016b).

384 Notably, the magnitude of increase in circulating follistatin after exercise in these previous 385 studies was considerably greater than observed in the present study. It is therefore possible that food consumption may have blunted the follistatin response after exercise in the present study. In particular, evidence suggests that insulin may inhibit follistatin production (Tao et al. 2018); including the response to exercise (Hansen et al. 2016b). Raised postprandial insulin may have blunted our ability to detect an effect of exercise intensity on circulating follistatin within our post-exercise observation period. An additional experiment is needed to test this hypothesis.

LECT2 is a novel hepatokine whose circulating levels are elevated in obesity, type 2 diabetes and non-alcoholic fatty liver disease (Okumura et al. 2013, Zhang et al. 2018). In vitro studies have shown that LECT2 triggers inflammation in vascular endothelium (Hwang et al. 2015) and directly inhibits insulin signalling in C2C12 myocytes (Lan et al. 2014) and 3T3-L1 adipocytes (Jung et al. 2018). It has therefore been suggested that LECT2 may provide a mechanistic link between NAFLD, T2DM and cardiovascular disease (Meex and Watt 2017).

397 In our previous research, 60 min of moderate-intensity treadmill exercise had no impact on 398 circulating LECT2 concentrations for up to $6 \mathrm{~h}$ post-exercise in healthy lean and overweight 399 men (Sargeant et al. 2018). Based on previous rodent data showing a negative regulatory 400 influence of hepatic AMPK-phosphorylation on LECT2 expression (Lan et al. 2014), we 401 hypothesised in the present study that high-intensity exercise would suppress circulating 402 LECT2. In contrast, we observed a significant increase in circulating LECT2 concentrations 403 immediately after both moderate- and high-intensity exercise; with no differences apparent in 
404 the hours thereafter. It must be noted, however, that statistical significance was lost at the $0 \mathrm{~h}$ 405 time point following adjustment for plasma volume changes, despite similar effect sizes (ES) 406 remaining (Cohen's $d$ before vs after plasma volume adjustment: $\mathrm{ES}=0.41$ vs $\mathrm{ES}=0.49$ and $407 \mathrm{ES}=0.42$ vs $\mathrm{ES}=0.45$ for $\mathrm{MOD}$ and $\mathrm{HIGH}$ compared to $\mathrm{CON}$, respectively). Therefore, 408 exercise-induced haemoconcentration may have contributed, in part, to the observed increase 409 in plasma LECT2 immediately after exercise.

410 Lan et al (2014) previously showed that hepatic LECT2 expression and circulating 411 concentrations were suppressed immediately after $3 \mathrm{~h}$ of treadmill running in $\mathrm{C} 57 \mathrm{BL} / 6 \mathrm{~J}$ mice.

412 This contrasting finding with our work may be related to species differences but may also be 413 due to the nature of the exercise utilised and/or the study participants. Specifically, given the 414 negative regulatory influence of hepatic AMPK, longer duration exercise with a greater 415 metabolic challenge may be needed to suppress LECT2 in humans. It is also possible that this 416 response may only occur in individuals with obesity in which circulating LECT2 is elevated; 417 however additional studies in populations with obesity are needed to test these hypotheses.

418 A key strength of our study is the precision with which exercise-related energy expenditure 419 was matched. Conversely, a limitation of the present study is that the observed hepatokine 420 responses occurred in a population of young, healthy men. Circulating levels of hepatokines 421 are modulated by adiposity and associated metabolic disease and, therefore, we cannot be 422 certain that our findings translate to different populations. The therapeutic benefits of exercise 423 are greater in clinical populations and therefore additional studies should investigate the 424 influence of exercise of varied intensities and durations in individuals with obesity and 425 associated metabolic disease.

426 In conclusion, this study demonstrates that circulating concentrations of FGF21 and follistatin 427 are transiently increased in response to acute bouts of aerobic exercise, whereas circulating 20 
428 LECT2 concentrations may not be sensitive to acute exercise stimuli. Furthermore, exercise 429 increases circulating FGF21 in an intensity-dependent manner, whilst the exercise-induced 430 increase in circulating follistatin may be independent of exercise intensity. These findings 431 support the notion that FGF21 and follistatin may contribute to the favourable metabolic effects 432 of exercise and a dose-dependent increase in FGF21 secretion may be partly responsible for 433 the additional benefits of high-intensity exercise. Additionally, these data extend our 434 understanding of the acute regulation of hepatokines in humans and may help to inform future 435 therapeutic interventions targeted at manipulating hepatokine concentrations. 
436 Conflict of interest statement

437 The authors declare no conflicts of interest.

438

439 Acknowledgements

440 The research was funded by the National Institute for Health Research (NIHR) Leicester and

441 Nottingham Biomedical Research Centres. The views expressed are those of the authors and

442 not necessarily those of the NHS, the NIHR or the Department of Health and Social Care. 


\section{References}

444 Alonge, K.M., Meares, G.P., and Hillgartner, F.B. 2017. Glucagon and insulin cooperatively

445 stimulate fibroblast growth factor 21 gene transcription by increasing the expression of

446 activating transcription factor 4. J. Biol. Chem. 292(13): 5239-5252.

447 doi:10.1074/jbc.M116.762922.

448 Amthor, H., Nicholas, G., McKinnell, I., Kemp, C.F., Sharma, M., Kambadur, R., et al. 2004.

449 Follistatin complexes myostatin and antagonises myostatin-mediated inhibition of

450 myogenesis. Dev. Biol. 270(1): 19-30. doi:10.1016/j.ydbio.2004.01.046.

451 Arafat, A.M., Kaczmarek, P., Skrzypski, M., Pruszyńska-Oszmalek, E., Kołodziejski, P., 452 Szczepankiewicz, D., et al. 2013. Glucagon increases circulating fibroblast growth factor 21

453 independently of endogenous insulin levels: a novel mechanism of glucagon-stimulated 454 lipolysis? Diabetologia. 56(3): 588-597. doi:10.1007/s00125-012-2803-y.

455 BonDurant, L.D., and Potthoff, M.J. 2018. Fibroblast growth factor 21: A versatile regulator 456 of metabolic homeostasis. Annu. Rev. Nutr. 38: 173-196. doi:10.1146/annurev-nutr-071816457064800.

458 Borg, G.A. 1973. Perceived exertion: a note on "history" and methods. Med. Sci. Sports. $459 \quad$ 5(2): 90-93.

460 Bredin, S.S., Gledhill, N., Jamnik, V.K., and Warburton, D.E. 2013. PAR-Q+ and ePARmed$461 \quad \mathrm{X}+$ : new risk stratification and physical activity clearance strategy for physicians and patients 462 alike. Can. Fam. Physician. 59(3): 273-277.

463 Chavez, A.O., Molina-Carrion, M., Abdul-Ghani, M.A., Folli, F., Defronzo, R.A., and 464 Tripathy, D. 2009. Circulating fibroblast growth factor-21 is elevated in impaired glucose 
465 tolerance and type 2 diabetes and correlates with muscle and hepatic insulin resistance.

466 Diabetes Care 32(8): 1542-1546. doi:10.2337/dc09-0684.

467 Cyphert, H.A., Alonge, K.M., Ippagunta, S.M., and Hillgartner, F.B. 2014. Glucagon

468 stimulates hepatic FGF21 secretion through a PKA- and EPAC-dependent posttranscriptional 469 mechanism. PLoS One. 9(4): e994996. doi: 10.1371/journal.pone.0094996.

470 Dill, D.B., and Costill, D.L. 1974. Calculation of percentage changes in volumes of blood, 471 plasma, and red cells in dehydration. J. Appl. Physiol. 37(2): 247-248.

472 doi:10.1152/jappl.1974.37.2.247.

473 Dushay, J., Chui, PC., Gopalakrishnan, G.S., Varela-Rey, M., Crawley, M., Fisher, F.M., et 474 al. 2010. Increased fibroblast growth factor 21 in obesity and non-alcoholic fatty liver 475 disease. Gastroenterology. 139(2): 456-463. doi:10.1053/j.gastro.2010.04.054.

476 Field, A. 2009. Discovering Statistics using SPSS, edn 3. London, UK: Sage.

477 Frayn, K.N. 1983. Calculation of substrate oxidation rates in vivo from gaseous exchange. J. 478 Appl. Physiol. Respir. Environ. Exerc. Physiol. 55(2): 628-634.

479 doi:10.1152/jappl.1983.55.2.628.

480 Fu, S., Fan, J., Blanco, J., Giminez-Cassina, A., Danial, N.N., Watkins, S.M., et al. 2012.

481 Polysome profiling in liver identifies dynamic regulation of endoplasmic reticulum

482 translatome by obesity and fasting. PLoS Genet. 8(8): e1002902.

483 doi:10.1371/journal.pgen.1002902.

484 Gälman, C., Lundåsen, T., Kharitonenkov, A., Bina, H.A., Eriksson, M., Hafström, I., et al. 485 2008. The circulating metabolic regulator FGF21 is induced by prolonged fasting and 486 PPARalpha activation in man. Cell Metab 8(2): 169-174. doi:10.1016/j.cmet.2008.06.014. 
487

488

489

490

491

492

493

494

495

496

497

498

499

500

501

502

Hansen, J.S., Pedersen, B.K., Xu, G., Lehmann, R., Weigert, C., and Plomgaard, P. 2016 b.

503 Exercise-induced secretion of FGF21 and follistatin are blocked by pancreatic clamp and

504 impaired in type 2 diabetes. J. Clin. Endocrinol. Metab. 101(7): 2816-2825.

505 doi:10.1210/jc.2016-1681.

506 Hayashi, Y., Nagasaka, S., Takahashi, N., Kusaka, I., Ishibashi, S., Numao, S., et al. 2005. A 507 single bout of exercise at higher intensity enhances glucose effectiveness in sedentary men. J. 508 Clin. Endocrinol. Metab. 90(7): 4035-4040. doi:10.1210/jc.2004-2092. 
509 Hwang, H.J., Jung, T.W., Hong, H.C., Seo, J.A., Kim, S.G., Kim, N.H., et al. 2015. LECT2

510 induces atherosclerotic inflammatory reaction via CD209 receptor-mediated JNK

511 phosphorylation in human endothelial cells. Metabolism 64(9): 1175-1182.

512 doi:10.1016/j.metabol.2015.06.001.

513 Inagaki, T., Dutchak, P., Zhao, G., Ding, X., Gautron, L., Parameswara, V., et al. 2007.

514 Endocrine regulation of the fasting response by PPARalpha-mediated induction of fibroblast

515 growth factor 21. Cell. Metab. 5(6): 415-425. doi:10.1016/j.cmet.2007.05.003.

516 Iroz, A., Couty, J.P., and Postic, C. 2015. Hepatokines: unlocking the multi-organ network in 517 metabolic diseases. Diabetologia. 58(8): 1699-1703. doi:10.1007/s00125-015-3634-4.

518 Jung, T.W., Chung, Y.H., Kim, H.C., Abd El-Aty, A.M., and Jeong, J.H. 2018. LECT2

519 promotes inflammation and insulin resistance in adipocytes via P38 pathways. J. Mol.

520 Endocrinol. 61(1): 37-45. doi:10.1530/JME-17-0267.

521 Kharitonenkov, A., Shiyanova, T.L., Koester, A., Ford, A.M., Micanovic, R., Galbreath, E.J., 522 et al. 2005. FGF-21 as a novel metabolic regulator. J. Clin. Invest. 115(6): 1627-1635. doi: $52310.1172 / \mathrm{JCI} 23606$.

524 Kim, K.H., Kim, S.H., Min, Y.K., Yang, H.M., Lee, J.B., and Lee, M.S. 2013. Acute exercise 525 induces FGF21 expression in mice and in healthy humans. PLoS One. 8(5): e63517. 526 doi:10.1371/journal.pone.0063517.

527 Kim, T., Holleman, C.L., Nason, S., Arble, D.M., Ottaway, N., Chabenne, J., et al. 2018. 528 Hepatic glucagon-receptor signalling enhances insulin-stimulated glucose disposal in rodents. 529 Diabetes. 67(11): 2157-2166. doi:10.2337/db18-0068. 
530 Kirpich, I.A., Gobejishvili, L.N., Bon Homme, M., Waigel, S., Cave, M., Arteel, G., et al.

531 2011. Integrated hepatic transcriptome and proteome analysis of mice with high-fat diet-

532 induced non-alcoholic fatty liver disease. J. Nutr. Biochem. 22(1): 38-45.

533 doi:10.1016/j.jnutbio.2009.11.009.

534 Kondo, H., Hojo, Y., Saito, T., Ikemoto, T., Katsuki, T., Shimada, K., et al. 2011.

535 Relationship between fibroblast growth factor 21 and extent of left ventricular remodelling

536 after acute myocardial infarction. J. Clinic. Experiment. Cardiol. 2(6) 1000139.

537 doi:10.4172/2155-9880.1000139.

538 Laeger, T., Henagan, T.M., Albarado, D.C., Redman, L.M., Bray, G.A., Noland, R.C., et al.

539 2014. FGF21 is an endocrine signal of protein restriction. J. Clin. Invest. 124(9): 3913-3922.

540 doi:10.1172/JCI74915.

541 Lan, F., Misu, H., Chikamoto, K., Takayama, H., Kikuchi, A., Mohri, K., et al. 2014. LECT2

542 functions as a hepatokine that links obesity to skeletal muscle insulin resistance. Diabetes.

543 63(5): 1649-1664. doi:10.2337/db13-0728.

544 Loyd, C., Magrisso, I.J., Haas, M., Balusu, S., Krishna, R., Itoh, N., et al. 2016. Fibroblast

545 growth factor 21 is required for beneficial effects of exercise during chronic high-fat feeding.

546 J. Appl. Physiol. 121(3): 687-698. doi:10.1152/japplphysiol.00456.2016.

547 Li, H., Wu, G., Fang, Q., Zhang, M., Hui, X., Sheng, B., et al. 2018. Fibroblast growth factor

54821 increases insulin sensitivity through specific expansion of subcutaneous fat. Nat.

549 Commun. 9(1): 272. doi:10.1038/s41467-017-02677-9.

550 Lira, F.S., Carnevali Jr, L.C., Zanchi, N.E., Santos, R.V.T., Lavoie, J.M., and Seelaender, M.

551 2012. Exercise intensity modulation of hepatic lipid metabolism. J. Nutr. Metab. 809576.

552 doi:10.1155/2012/809576.

27 
553 Markan, K.R., Naber, M.C., Ameka, M.K., Anderegg, M.D., Mangelsdorf, D.J., Kliewer, 554 S.A., et al. 2014. Circulating FGF21 is liver derived and enhances glucose uptake during 555 refeeding and overfeeding. Diabetes. 63(12): 4057-4063. doi:10.2337/db14-0595.

556 Meex, R.C.R., and Watt, M.J. 2017. Hepatokines: linking non-alcoholic fatty liver disease 557 and insulin resistance. Nat. Rev. Endocrinol. 13(9): 509-520. doi:10.1038/nrendo.2017.56.

558 Meex, R.C.R., Hoy, A.J., Morris, A., Brown, R.D., Lo, J.C., Burke, M., et al. 2015. Fetuin B 559 is a secreted hepatocyte factor linking steatosis to impaired glucose metabolism. Cell. Metab. 560 22(6): 1078-1089. doi:10.1016/j.cmet.2015.09.023.

561 Mifflin, M.D., St Jeor, S.T., Hill, L.A., Scott, B.J., Daugherty, S.A., and Koh, Y.O. 1990. A 562 new predictive equation for resting energy expenditure in healthy individuals. Am. J. Clin. 563 Nutr. 51(2): 241-247. doi:10.1093/ajcn/51.2.241.

564 Okumura, S., Unoki-Kubota, H., Matsushita, Y., Shiga, T., Moriyoshi, Y., Yamagoe, S. et al. 565 2013. Increased serum leukocyte cell-derived chemotaxin 2 (LECT2) levels in obesity and 566 fatty liver. Biosci. Trends. 7(6): 276-283.

567 Perakakis, N., Mougios, V., Fatouros, I., Siopi, A., Draganidis, D., Peradze, N., et al. 2018.

568 Physiology of activins/follistatins: associations with metabolic and anthropometric variables 569 and response to exercise. J. Clin. Endocrinol. Metab. 103(10): 3890-3899.

570 doi:10.1210/jc.2018-01056.

571 Potthoff, M.J., Inagaki, T., Satapati, S., Ding, X., He, T., Goetz, R., et al. 2009. FGF21

572 induces PGC-1alpha and regulates carbohydrate and fatty acid metabolism during the

573 adaptive starvation response. Proc. Natl. Acad. Sci. U. S. A. 106(26): 10853-10858.

574 doi:10.1073/pnas.0904187106. 
575 Robergs, R.A., Dwyer, D., and Astorino, T. 2010. Recommendations for improved data 576 processing from expired gas analysis indirect calorimetry. Sports. Med. 40(2): 95-111.

577 doi:10.2165/11319670-000000000-00000.

578 Rynders, C.A., Weltman, J.Y., Jiang, B., Breton, M., Patrie, J., Barrett, E.J., et al. 2014.

579 Effects of exercise intensity on postprandial improvement in glucose disposal and insulin

580 sensitivity in prediabetic adults. J. Clin. Endocrinol. Metab. 99(1): 220-228.

581 doi:10.1210/jc.2013-2687.

582 Sargeant, J.A., Aithal, G.P., Takamura, T., Misu, H., Takayama, H., Douglas, J.A., et al. 583 2018. The influence of adiposity and acute exercise on circulating hepatokines in normal584 weight and overweight/obese men. Appl. Physiol. Nutr. Metab. 43(5): 482-490. 585 doi:10.1139/apnm-2017-0639.

586 Tao, R., Wang, C., Stöhr, O., Qiu, W., Hu, Y., Miao, J., et al. 2018. Inactivating hepatic 587 follistatin alleviates hyperglycaemia. Nat. Med. 24(7): 1058-1069. doi:10.1038/s41591-018$588 \quad 0048-0$.

589 Toye, A.A., Dumas, M.E., Blancher, C., Rothwell, A.R., Fearnside, J.F., Wilder, S.P., et al. 590 2007. Subtle metabolic and liver gene transcriptional changes underlie diet-induced fatty liver 591 susceptibility in insulin-resistant mice. Diabetologia. 50(9): 1867-1879. doi:10.1007/s00125$592 \quad 007-0738-5$.

593 Wahren, J., Felig, P., and Hagenfeldt, L. 1978. Physical exercise and fuel homeostasis in 594 diabetes mellitus. Diabetologia. 14(4): 213-222.

595 Weigert, C., Hoene, M., and Plomgaard, P. 2018. Hepatokines - a novel group of exercise 596 factors. Pflügers. Arch. doi:10.1007/s00424-018-2216-y. [Epub ahead of print]. 
597 Wen, C.P., Wai, J.P., Tsai, M.K., Yang, Y.C., Cheng, T.Y., Lee, M.C., et al. 2011. Minimum 598 amount of physical activity for reduced mortality and extended life expectancy: a prospective 599 cohort study. Lancet. 378(9798): 1244-1253. doi:10.1016/S0140-6736(11)60749-6.

600 Wolfe, R.R., Nadel E.R., Shaw, J.H., Stephenson, L.A., and Wolfe, M.H. 1986. Role of 601 changes in insulin and glucagon in glucose homeostasis in exercise. J. Clin. Invest. 77(3): 602 900-907. doi:10.1172/JCI112388.

603 Xu, J., Stanislaus, S., Chinookoswong, N., Lau, Y.Y., Hager, T., Patel, J., et al. 2009. Acute 604 glucose-lowering and insulin-sensitizing action of FGF21 in insulin-resistant mouse models-605 association with liver and adipose tissue effects. Am. J. Physiol. Endocrinol. Metab. 297(5): 606 E1105-E1114. doi:10.1152/ajpendo.00348.2009.

607 Yaden, B.C., Croy, J.E., Wang, Y., Wilson, J.M., Datta-Mannan, A., Shetler, P., et al. 2014. 608 Follistatin: a novel therapeutic for the improvement of muscle regeneration. J. Pharmacol. 609 Exp. Ther. 349(2): 355-371. doi:10.1124/jpet.113.211169.

610 Zhang, Z., Zeng, H., Lin, J., Hu, Y., Yang, R., Sun, J., et al. 2018. Circulating LECT2 levels 611 in newly diagnosed type 2 diabetes mellitus and their association with metabolic parameters: 612 An observational study. Medicine. 97(15): e0354. doi:10.1097/MD.0000000000010354.

613 Zhao, C., Qiao, C., Tang, R.H., Jiang, J., Li, J., Martin, C.B., et al. 2015. Overcoming insulin 614 insufficiency by forced follistatin expression in $\beta$-cells of $\mathrm{db} / \mathrm{db}$ mice. Mol. Ther. 23(5): 866615 874. doi:10.1038/mt.2015.29. 


\section{Tables}

617 Table 1. Participant characteristics $(n=10)$.

\begin{tabular}{lc}
\hline \multicolumn{1}{c}{ Characteristic } & Mean $\pm \mathrm{SD}$ \\
\hline Age (years) & $26 \pm 2$ \\
Height $(\mathrm{m})$ & $1.77 \pm 0.07$ \\
Body mass $(\mathrm{kg})$ & $80.2 \pm 6.2$ \\
BMI $\left(\mathrm{kg} \cdot \mathrm{m}^{-2}\right)$ & $25.6 \pm 1.7$ \\
Waist circumference $(\mathrm{cm})$ & $83.7 \pm 4.1$ \\
Body fat $(\%)$ & $15.4 \pm 4.4$ \\
VO $_{2}$ peak $\left(\mathrm{mL} \cdot \mathrm{kg}^{-1} \cdot \mathrm{min}^{-1}\right)$ & $49.8 \pm 5.3$ \\
\hline
\end{tabular}

Note: BMI, body mass index; $\dot{\mathrm{V}} \mathrm{O}_{2}$ peak, peak oxygen uptake. 
619 Table 2. Exercise characteristics $(n=10)$.

\begin{tabular}{|c|c|c|c|c|c|c|c|}
\hline \multirow[b]{2}{*}{ Treadmill speed $\left(\mathrm{km} \cdot \mathrm{h}^{-1}\right)$} & \multicolumn{3}{|c|}{ MOD } & \multicolumn{3}{|c|}{ HIGH } & \multirow{2}{*}{$\begin{array}{c}P \text {-value } \\
<\mathbf{0 . 0 0 1}\end{array}$} \\
\hline & 8.2 & \pm & 1.4 & 11.6 & \pm & 1.6 & \\
\hline Oxygen uptake $\left(\mathrm{L} \cdot \mathrm{min}^{-1}\right)$ & 2.17 & \pm & 0.26 & 2.98 & \pm & 0.37 & $<0.001$ \\
\hline Exercise intensity $\left(\% \dot{\mathrm{V}}_{2}\right.$ peak $)$ & 54.6 & \pm & 1.7 & 74.7 & \pm & 1.8 & $<0.001$ \\
\hline Exercise duration (min) & 57 & \pm & 8 & 42 & \pm & 6 & $<0.001$ \\
\hline Gross energy expenditure $(\mathrm{kJ})$ & 2475 & \pm & 70 & 2488 & \pm & 58 & 0.459 \\
\hline Respiratory exchange ratio & 0.90 & \pm & 0.03 & 0.99 & \pm & 0.03 & $<0.001$ \\
\hline Carbohydrate oxidation $(\%)$ & 67 & \pm & 10 & 94 & \pm & 8 & $<\mathbf{0 . 0 0 1}$ \\
\hline Fat oxidation $(\%)$ & 33 & \pm & 10 & 6 & \pm & 8 & $<0.001$ \\
\hline Heart rate $\left(\right.$ beats $\cdot \mathrm{min}^{-1}$ ) & 142 & \pm & 10 & 168 & \pm & 9 & $<\mathbf{0 . 0 0 1}$ \\
\hline Rating of perceived exertion & 11 & \pm & 2 & 16 & \pm & 1 & $<\mathbf{0 . 0 0 1}$ \\
\hline
\end{tabular}

Note: Data are presented as means $\pm \mathrm{SD} . \dot{\mathrm{V}}_{2}$ peak, peak oxygen uptake. 
621 Table 3. Area under the hepatokine concentration vs time curve during CON, MOD and

622 HIGH trials for the preprandial (BL-2 h), postprandial $(2-7 \mathrm{~h})$ and total trial $(8 \mathrm{~h})$ periods.

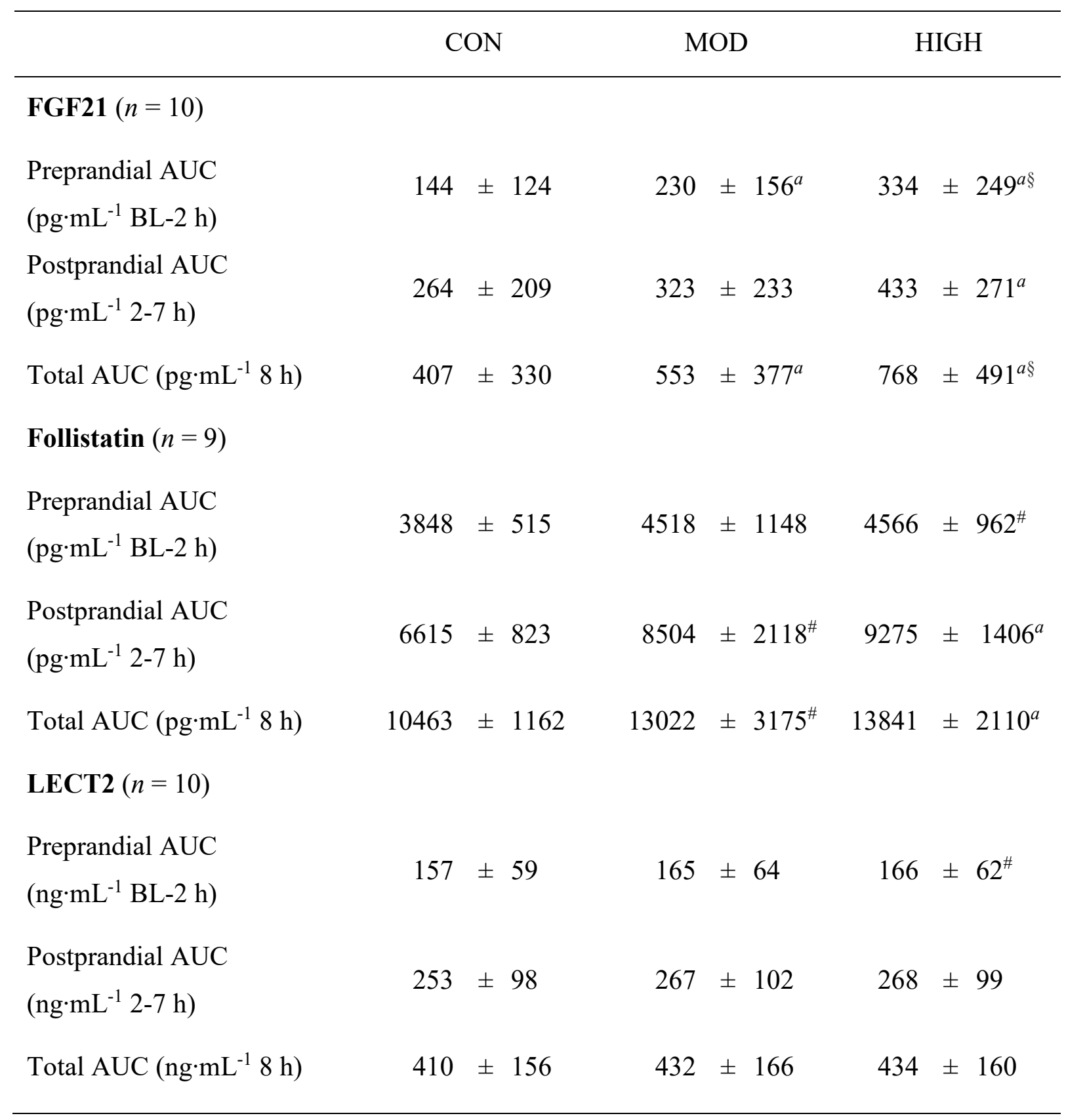

623 Note: Data are presented as means \pm SD. FGF21, fibroblast growth factor 21; AUC, area 624 under curve; BL, baseline; LECT2, leukocyte cell-derived chemotaxin 2.

$625{ }^{a}$ Significantly different from $\operatorname{CON}(P<0.05)$.

$626 \quad{ }^{\#}$ Tended to differ from $\operatorname{CON}(P<0.07)$.

627 §Tended to differ from MOD $(P<0.07)$. 
629 Figure 1. Schematic illustration of the main trial protocol. Duration of exercise in MOD and $630 \mathrm{HIGH}=57 \pm 8$ and $42 \pm 6$ min, respectively. Participants were provided with $35 \%$ of 631 estimated daily energy requirements at both $2 \mathrm{~h}$ and $6 \mathrm{~h}$.

632

633 Figure 2. Plasma concentrations of fibroblast growth factor 21 (FGF21) (A), follistatin (B)

634 and leukocyte cell-derived chemotaxin 2 (LECT2) (C) during the control (open circles),

635 moderate-intensity exercise (black circles) and high-intensity exercise (grey circles) trials.

636 Data are presented as means \pm SEM, $n=10$ for FGF2 1 and LECT2 data, and $n=9$ for

637 follistatin data. Black rectangle indicates moderate-intensity exercise, grey rectangle indicates

638 high-intensity exercise and hatched rectangles indicate test meals. BL, baseline. ${ }^{a} \mathrm{MOD}$

639 significantly different from $\mathrm{CON}(P<0.05) ;{ }^{b} \mathrm{HIGH}$ significantly different from $\mathrm{CON}(P<$ 640 0.05); ${ }^{c} \mathrm{HIGH}$ significantly different from $\operatorname{MOD}(P<0.05)$.

641

642 Figure 3. Plasma concentrations of glucose (A), non-esterified fatty acids (NEFA) (B),

643 glucagon (C), insulin (D) and the glucagon to insulin ratio (E) during the control (open

644 circles), moderate-intensity exercise (black circles) and high-intensity exercise (grey circles)

645 trials. Data are presented as means \pm SEM. Black rectangle indicates moderate-intensity

646 exercise, grey rectangle indicates high-intensity exercise and hatched rectangles indicate test

647 meals. BL, baseline. ${ }^{a}$ MOD significantly different from CON $(P<0.05) ;{ }^{b} \mathrm{HIGH}$ significantly

648 different from $\mathrm{CON}(P<0.05)$; ${ }^{c} \mathrm{HIGH}$ significantly different from $\mathrm{MOD}(P<0.05)$. 
651 Fig 1

652

653

654

655

656

657

658

659

I

660

661

Blood

Time (h)

(Time of day)

662

Standardised meal

663

664

665

666

667

668

669

670

671

672

673

674

675

676 
$677 \quad$ Fig 2

A

$a, b, c$

678
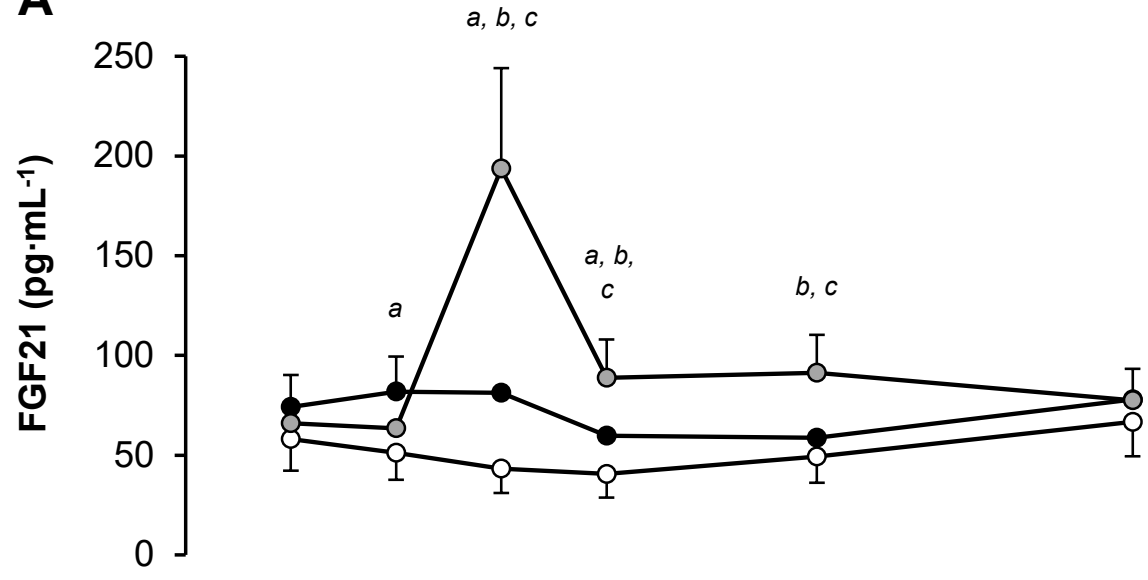

B

679

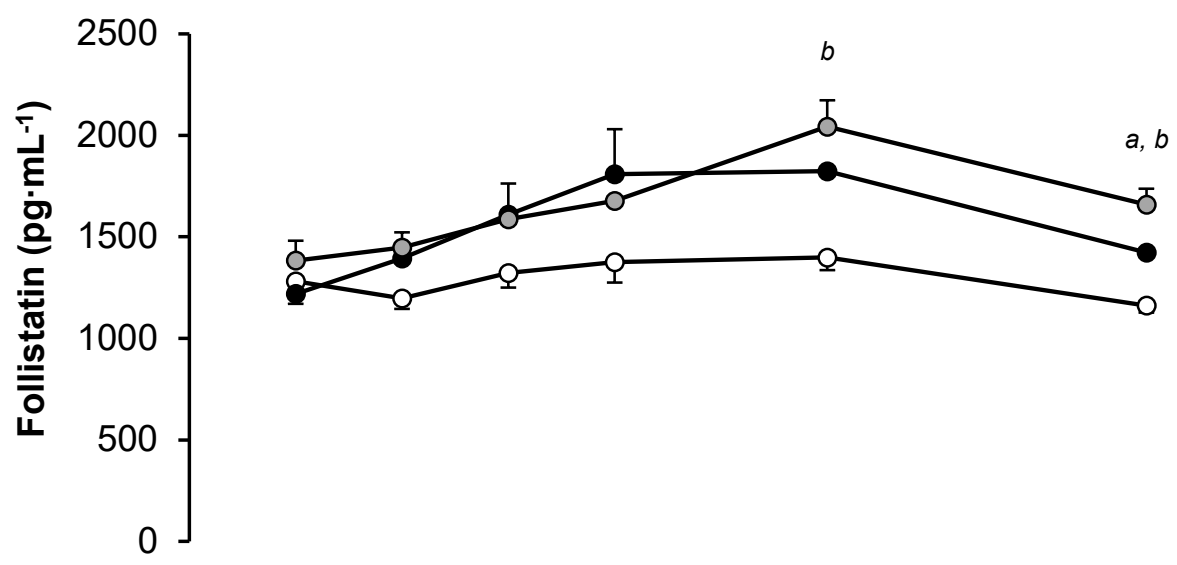

C

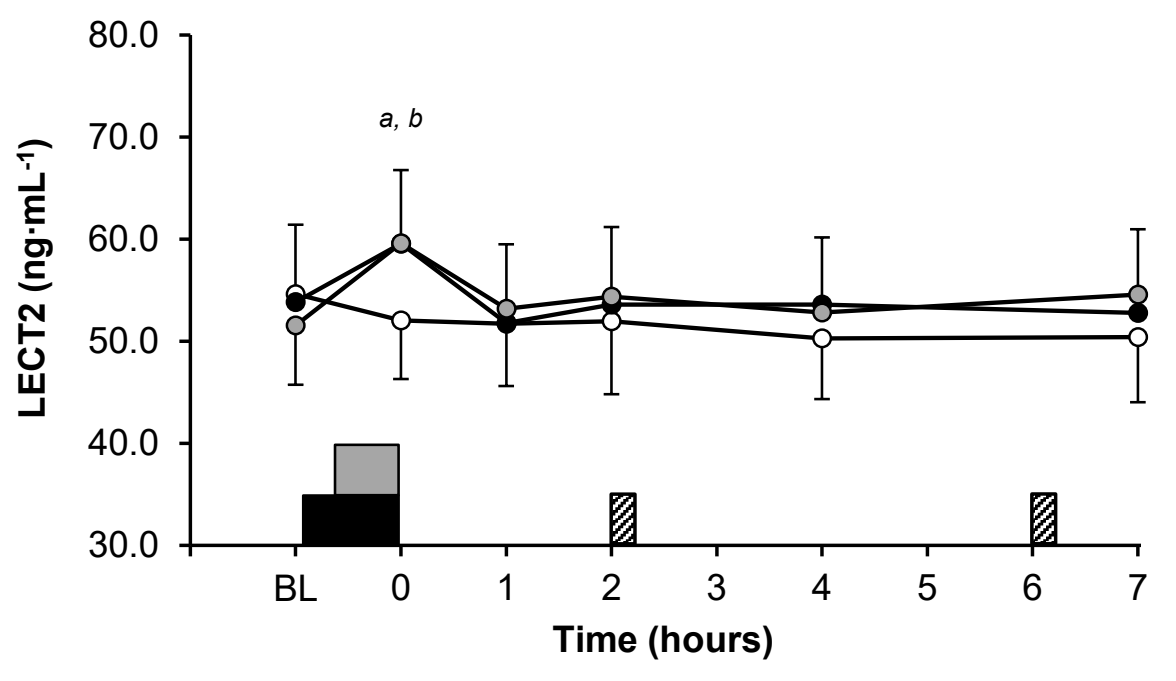

680

681

682

683

684 
$685 \quad$ Fig 3

686

687

688
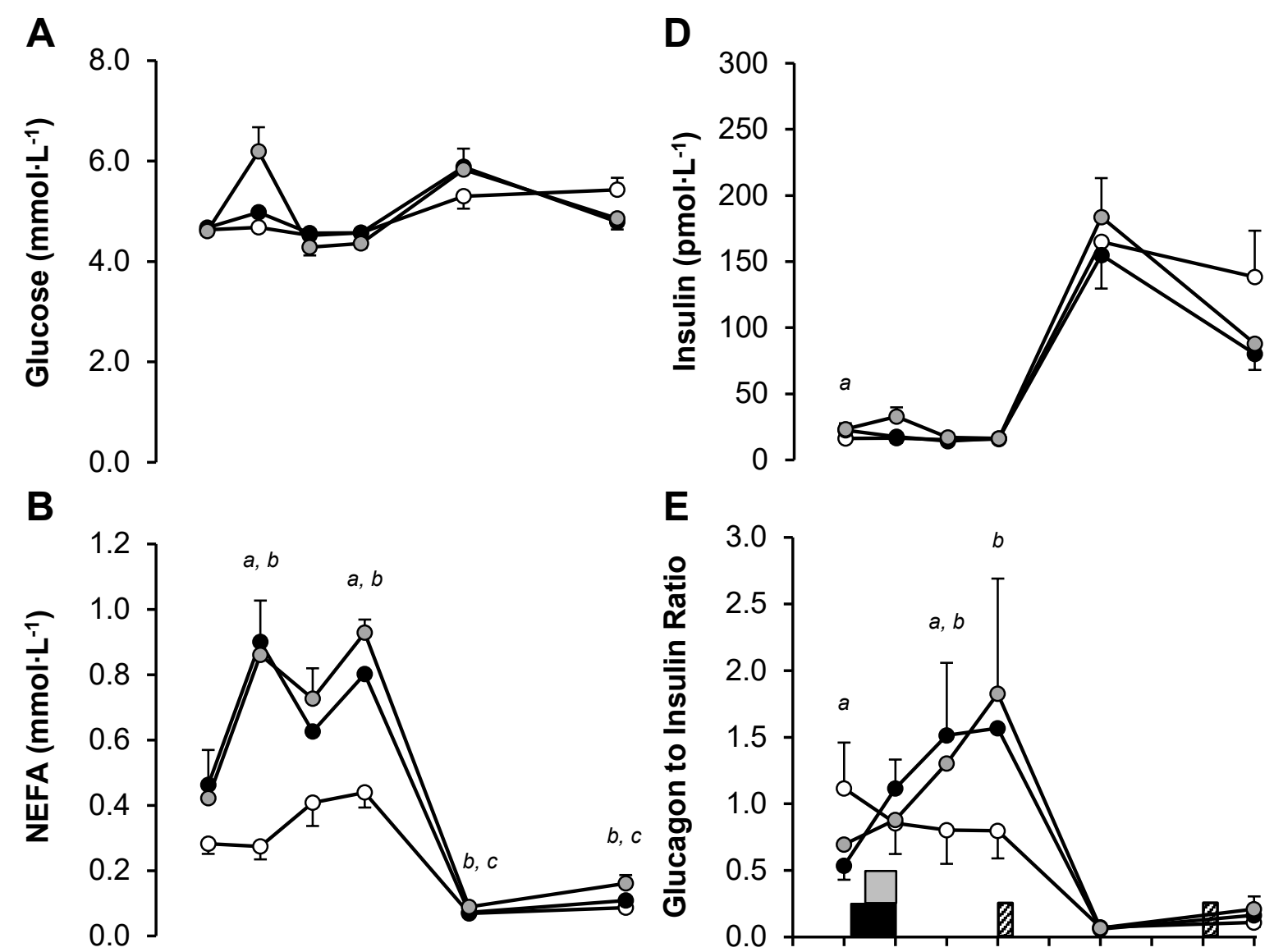

689

C
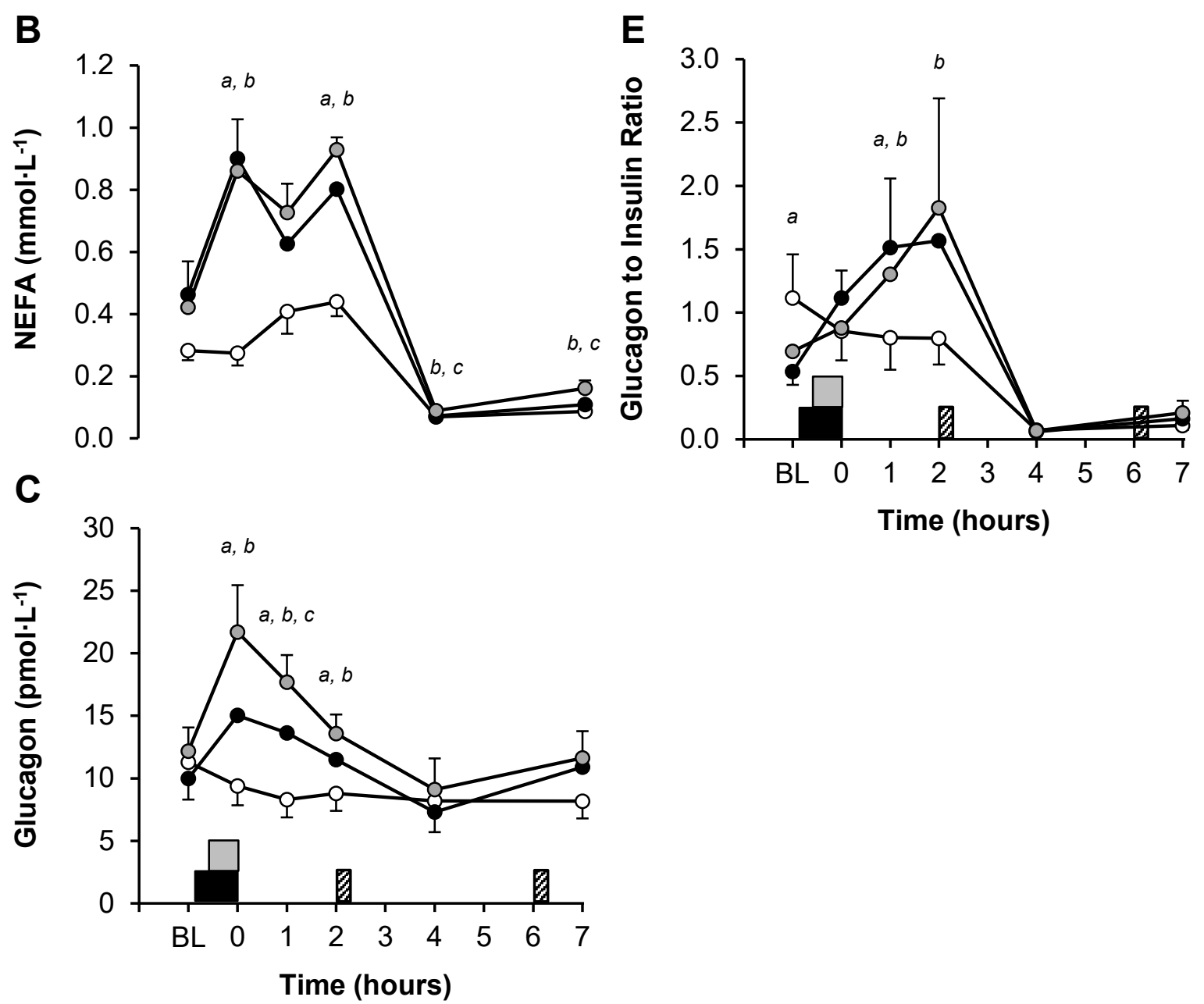

Time (hours)

690

691

692

693 